\title{
Los textos colombinos a la luz del discurso de Bartolomé de Las Casas
}

\section{The columbian texts in the light of Bartolomé de Las Casas's discourse}

Danuta Teresa MozejKo

\author{
Universidad Nacional de Córdoba. Córdoba, Argentina. \\ cdrcosta@ciudad.com.ar
}

\section{RESUMEN}

Si bien nadie desconoce que los textos supuestamente producidos por Colón durante los viajes del descubrimiento se conservan gracias a la versión que de ellos ofrecen los escritos lascasianos, particularmente la Historia de las Indias, las lecturas y re-escrituras de los diarios de viaje se fundan en una representación del padre Las Casas como fiel y preciso transcriptor de los relatos colombinos. Restituir los textos del Almirante al intertexto que los engloba implica tener en cuenta el proyecto del agente que produce este último y, en consecuencia, focalizar en operaciones de selección, organización y énfasis particulares que, más que transparentar las versiones primeras, las cargan de sentidos nuevos. Desde esta perspectiva, el registro de los acontecimientos ocurridos durante el viaje de Colón puede ser entendido como una serie de secuencias que articulan el mandato según normas incuestionables, la culpa y el castigo de un personaje que, consciente de su misión, la transforma en un pecado que debe expiar.

Palabras claves: Intertextualidad, paraíso, hombre natural, culpa.

\section{ABSTRACT}

Although it is well known that the texts presumably produced by Columbus during his discovery voyages remain thanks to the version of them offered in Las Casas's writings, in particular Historia de las Indias, the readings and re-writings of the travel diaries are based on a representation of Father Las Casas as a faithful and accurate transcriber of the 
Columbian accounts. Restoring the Admiral's texts to the intertext that embraces them involves bearing in mind the project of the agent producing the latter and therefore focusing on particular operations of selection, organization and emphasis which, rather than make the earlier versions more transparent, infuse them with new meanings. From this perspective, the record of the events that occurred during Columbus's voyage may be understood as a series of sequences articulating the mandate according to unquestionable norms, the guilt and the punishment of a character who, aware of his mission, turns it into a sin he must atone for.

Keywords: Intertextuality, paradise, natural man, guilt.

Recibido: 15-07-2009.

Aceptado: 28-09-2009.

\section{INTRODUCCIÓN}

T

a incorporación de un texto en otro en cualquiera de sus formas, implica subordinar los efectos de sentido del primero a la intencionalidad ${ }^{1}$ que rige al segundo. En el caso que nos ocupa, no es posible pensar en los relatos de viaje de Cristóbal Colón, especialmente el primero y el tercero, sin tener en cuenta los objetivos buscados por su transcriptor, fray Bartolomé de las Casas. Sin embargo, tanto en las lecturas críticas ${ }^{2}$ como en las re-escrituras ficcionales ${ }^{3}$ de los diarios del Almirante se ha insistido en la autoría de Colón como si fuera el responsable único de las versiones que llegan hasta nosotros.

No pretendemos abordar los textos desde la perspectiva de una crítica genética, imposible en tanto no contamos con las versiones originales de los escritos colombinos, pero sí creemos que pueden leerse señalando aquellos aspectos que se vinculan con los objetivos de la obra lascasiana, sin que esto implique negar que, en sus formas originarias, los discursos del Almirante hayan podido contener los rasgos que luego rescata Las Casas. De todas formas, su conservación sobre todo en la Historia de las Indias, las repeticiones y acentuaciones de las

\footnotetext{
${ }^{1}$ Entendemos intencionalidad como una orientación general de la práctica, en este caso, discursiva, sin que ésta sea necesariamente consciente y menos aún, necesariamente eficaz. En el caso específico del padre Las Casas, sin embargo, bien podría pensarse en términos de intención ya que los objetivos de su escritura -al menos los que nos interesan en este trabajo- se encuentran explicitados en el texto.

${ }^{2}$ A título de ejemplo, podemos citar: O’Gorman (1984); Todorov (1987); Pastor (1988); Sánchez Macgrégor (1991); Jitrik (1992); González Ortega (2006).

${ }^{3}$ Pensamos en El arpa y la sombra (1979) de Carpentier, Vigilia del Almirante (1992) de Roa Bastos, entre otros.
} 
que son objeto, darían cuenta de las opciones realizadas por el fraile dominico sobre la base de los textos del descubridor. Como sostiene Margarita Zamora:

A través de sus intervenciones editoriales Las Casas no solamente se insinúa a sí mismo en el diario de Colón, toma posesión de él, adaptándolo a su propia intención semántica y expresiva, como sostiene Bakhtin [...] El Diario no es el diario de Colón, ni siquiera su equivalente; es su sustituto, un texto nuevo, el producto de la asimilación y transformación de las palabras de Colón por las de Las Casas (1993: 48-49).

[...] nuestra lectura de los textos colombinos fue completamente definida por la institucionalización de una ficción editorial que crea la ilusión del carácter prístino y de la absoluta autoridad de la voz de Colón al reforzar la total supresión o manipulación del comentario de Las Casas (1993: 72)

Partiremos de un breve esbozo del modo como, a nuestro juicio, Las Casas presenta la historia de Colón y el descubrimiento, para luego señalar aquellos elementos que resultan susceptibles de una lectura que proyecte la intencionalidad que rige el texto englobante sobre el texto incluido gracias a operaciones de selección, énfasis y organización de los fragmentos.

\section{LA PROPUESTA DE LAS CASAS EN LA HISTORIA DE LAS INDIAS}

Para el fraile dominico, Colón es el sujeto de una misión que consiste en ejecutar el mandato de Dios. El plan divino viene impuesto desde el nombre que recibe el navegante en el bautismo:

Suele la divina Providencia ordenar que se pongan nombres y sobrenombres a personas que señala para se servir conformes a los oficios que les determina cometer [...] Llamóse, pues, por nombres, Cristóbal, conviene a saber, Christum ferens, que quiere decir traedor o llevador de Cristo [...] (Lib.I, Cap. II, T. I, 28) .

O’Gorman sintetiza el objetivo del viaje de Colón, tal como lo plantea el fraile dominico:

${ }^{4}$ La traducción es nuestra.

${ }^{5}$ Citamos según la edición de 1981 que figura en la bibliografía, señalando el Libro, Capítulo, Tomo y número de página correspondientes. 
[...] si ha de decirse en verdad quién fue el descubridor de América, debe contestarse que fue Cristóbal Colón, pero no en virtud de los propósitos y convicciones personales que animaron su empresa, sino como instrumento elegido por la Providencia para realizar la trascendental hazaña. Y si ha de precisarse qué fue lo que descubrió, debe decirse, no que fueron tales o cuales regiones geográficamente determinadas, sino el oculto camino por donde llegaría Cristo a aquellos numerosos y olvidados pueblos para cosechar entre ellos el místico fruto de la salvación eterna (1984: 29).

En relación con su destino, Colón aparece como sujeto plenamente competente. Posee el don de la $\mathrm{fe}^{6}$, noble y antiguo linaje, las virtudes requeridas, pero sobre todo, conoce la misión encomendada y sabe que es posible realizarla. El héroe no suele realizar sus proezas por casualidad y tampoco resulta pensable que Dios le encomiende una misión imposible para la cual le falten competencias. Las Casas da cuenta explícita de la capacidad plena de Colón para el hacer encomendado, incluyendo la aceptación consciente y voluntaria de su destino que pone en boca del navegante en primera persona:

Y porque Dios le dotó de alto juicio, de gran memoria y de vehemente aficción, tratando muchas veces con hombres doctos, y con su infatigable trabajo estudioso, y principalmente, a lo que yo cierto puedo y debo conjeturar y aun creer, por la gracia singular que le concedió para el ministerio que le cometía, consiguió la médula y substancia necesaria de las otras ciencias [...] [...] de forma que me abrió Nuestro Señor el entendimiento con mano palpable, a que era hacedero navegar de aquí a las Indias, y me abrasó la voluntad para la ejecución dello [...] (Lib.I, Cap.III, T.I, 31).

Las Casas señala especialmente la competencia del navegante en el orden del saber referido a la misión: ésta consiste en transmitir la fe a los indígenas, se inscribe en el plan divino y están dadas todas las condiciones favorables para ejecutarla. Por ello, Las Casas rechaza la versión de Agustín Justiniano, quien alude al "oficio mecánico" (Lib.I, Cap.III, T.I, 33) quitándole, por lo mismo, toda responsabilidad y mérito al trabajo de Colón.

\footnotetext{
6 "En las cosas de la religión cristiana, sin duda era católico y de mucha devoción; cuasi en cada cosa que hacía y decía o quería comenzar a hacer, siempre anteponía: "En el nombre de la Santa Trinidad haré esto o verná esto [...]” (Lib. I, Cap.II, T.I, 29). De esta manera, Las Casas borra toda posibilidad de que Colón aparezca como carente del objeto a transmitir, por un lado, y elimina el riesgo de que se aluda a su posible condición de judío, sobre todo teniendo en cuenta la referencia a la Trinidad.
} 
El navegante ha de transmitir la fe a los indígenas, pero para ello debe proceder primero al descubrimiento, programa instrumental en relación al básico. Por otra parte, se enuncian otras tareas, fundamentalmente orientadas a la obtención de prestigio personal, poder y, sobre todo, riquezas, pero éstas han de estar subordinadas a la difusión de los valores cristianos.

El programa del descubrimiento se realiza según las pautas de la narración tradicional: Colón busca ayudantes, surgen distintos obstáculos de los que sale victorioso aunque algunos de sus colaboradores se transformen en oponentes como es el caso de los hermanos Pinzón. Uno de ellos, Martín Alonso, será luego el antihéroe que se hace pasar por primer descubridor para merecer así los beneficios reservados al héroe. El juicio sustanciado al regreso a España se convierte en la instancia de reconocimiento en el que se otorgan a Cristóbal Colón los honores y las mercedes prometidos, incluido el homenaje del enunciador quien, a partir de ese momento, considera necesario aludir al personaje agregándole el título merecidamente ganado: el Almirante.

Sin embargo, en lo que se refiere al programa principal, el héroe se aparta del plan divino. Poco a poco va reemplazando el valor principal, la fe, por los bienes económicos. Si bien éstos no carecen de importancia ya que "Aprovecha muy mucho [...] que el rey sea rico" (Lib.I, Cap. LXXVI, T.I, 330), no es lícito alterar la jerarquía de valores: las almas redimidas son "granos de trigo celestial", "piedras muy vivas" y no son comparables con el oro, la plata, las perlas o las piedras preciosas (Lib. I, Cap. LXXVI, T. I, 329). Colón sustituye los bienes máximos por los menores, con lo cual los valores espirituales y eternos son reemplazados por los materiales y terrenales, transitorios; los valores supremos puestos en juego con el descubrimiento, son desplazados por los temporales e individuales. El desvío de Colón consiste en buscar riquezas y prestigio personal, olvidando la dimensión trascendente involucrada en el mandato divino. Dada su competencia plena como sujeto de hacer consciente y voluntario, el Almirante se convierte en culpable de la transgresión a las normas establecidas. Estas normas, claramente explicitadas en el texto lascasiano, dependen de tres destinadores fundamentales:

a) Dios, quien ordena convertir a los indígenas dotados por El mismo de la competencia previa necesaria para la recepción de la fe:

[...] hallaremos por experiencia lo que la Escritura divina nos enseña acerca de la infalible providencia de Dios, conviene a saber, que uno de los principales cuidados que Dios tiene [...] es acerca de la prueba y de la guarda y conservación de la verdad [...] Por manera que para que esta verdad de ser estas gentes 
dóciles, pacíficas, benignas de su natural y aparejadas tan bien y muy más que otras para ser doctrinadas y acostumbradas en toda virtud oral, y, por consiguiente, capaces y fácilmente atraíbles a la fe católica y religión cristiana, si les es propuesta y predicada como Cristo lo estableció, y a todas las otras naciones del mundo la Iglesia universal la ha propuesto siempre y predicado [...] aquestas gentes no son otras sino aquellas que sucedieron de nuestro primer padre Adán, y esto basta para que con ellas se deban guardar los preceptos divinos y naturales y las reglas de caridad que han sido guardadas y usadas con nosotros [...] (Lib.I, Cap. CLXXIV, T. II, 163).

Por consiguiente, y siempre por mandato divino, se establece un principio de equivalencia entre españoles e indígenas: siendo todos hijos de Adán, solamente hay una diferencia de tiempos en el acceso a los valores trascendentes ya que los europeos han recibido la fe antes que los hombres a quienes deben convertir; pero por eso mismo, el proceso ha de realizarse mediante la persuasión que provoque en los destinatarios el querer adherir a los nuevos valores, con lo cual adquieren el carácter de sujetos voluntarios y conscientes en lugar de destinatarios pasivos, inconscientes e involuntarios de la imposición de los españoles. El no respeto de la condición humana del indígena convierte en pecado el ejercicio unilateral del poder por parte de los españoles.

b) La Corona que dicta leyes acordes con las divinas:

La razón clara lo muestra, que no se había de entrar de rondón ni como en su casa en estas tierras, ni en forma de guerra [...] como aún trajo en la instrucción y mandado que le dieron los reyes, y hacer todos cuantos comedimientos y tomar todos cuantos medios de paz y amor y dulzura y para evitar escándalo y turbación de los pusilos inocentes nos enseña y manda la suave ley evangélica [...] (Lib. I, Cap. XCIII, T.I, 381).

c) La Naturaleza, que también establece normas. Estas resultan particularmente importantes en el texto de Las Casas. En efecto, en la época no era verosímil una presentación del indígena como conocedor de las leyes emanadas del Dios cristiano y de la corona española, pero la ley natural era percibida como norma universalmente válida que regía a todos los seres humanos, cualquiera fuera la divinidad que reconocieran o el rey de quien fueran súbditos. Más aún, en la presentación que hace Las Casas del indígena americano, hay una particular insistencia en que no se le reconoce 
"secta"; esto lo haría pasible de la calificación de idólatra y, por lo mismo, vinculado con lo demoníaco, por lo cual su conversión hubiese resultado mucho más difícilmente pensable. El indígena, hijo de Adán, aparece como el hombre recién creado, habitante del paraíso y respetuoso de las leyes naturales ${ }^{7}$ :

Estas gentes [...] fueron sobre todas las destas Indias y creo sobre todas las del mundo, en mansedumbre, simplicidad, humildad, paz y quietud y en otras virtudes naturales, señaladas, que no parecía sino que Adán no había en ellas pecado (Lib. II, Cap. XLIII, T.II, 347, énfasis nuestro).

Por su parte, Colón aísla al indio de su espacio "natural", mata a señores "naturales", separa al marido de su mujer destruyendo la institución "natural" del matrimonio.

La violación de esta triple serie de normas configura la culpa y traslada a Colón y con él, a los españoles encargados de la conquista, del ámbito regido por los destinadores positivos tales como Dios, la Corona y la Naturaleza, al dependiente del demonio: "Esta invención comenzó aquí excogitada, inventada y rodeada por el diablo [...]" (Lib.I, Cap. CIV, T.I, 413). Por el contrario, el indígena, inscripto en el espacio regulado por las leyes naturales, se configura como el sujeto positivo, dispuesto a la conversión.

En el modo como es presentado el "natural" de América en el texto de Las Casas, hay un detalle no menor en tanto garantiza la condición humana de este personaje: en todas las caracterizaciones de los diferentes pueblos, el dominico insiste en dos atributos del indígena como son su capacidad para dominar la tierra haciéndola producir el alimento que él mismo necesita para su subsistencia y su capacidad para gobernar; con ello demuestra sus condiciones de ser racional susceptible de ejercer el poder sobre la naturaleza que lo rodea y la naturaleza humana, rasgos que demuestran su "humanidad".

\section{LOS TEXTOS DE COLÓN}

Dos géneros discursivos diferentes caracterizan los textos en los que se relatan el primero y tercer viajes de Colón. El primero se presenta en forma de diario de

\footnotetext{
${ }^{7}$ También la culpa del indígena, cuando sucede, se define en este marco. Así, por ejemplo, los indígenas que traicionan a su pueblo y ayudan a los conquistadores violan la ley natural: "[...] este rey Guacanagarí ofendía y violaba mucho la ley natural [...] y pecaba mortalmente ayudando y manteniendo, favoreciendo y conservando a los cristianos [...]” (Lib. I, Cap. CII, T.I, 404).
} 
viaje, sintetizado por Las Casas en tercera persona singular con algunas incorporaciones del discurso directo del Almirante, pero manteniendo la ilusión de cierta fidelidad al texto de origen. El tercer viaje es narrado en primera persona, en una supuesta carta dirigida por el mismo Colón a los reyes y que Las Casas transcribe; si bien puede suponerse la existencia de un diario pormenorizado, no hay rastros de este documento y sólo se conserva la transcripción de una síntesis.

\subsection{DIARIO DEL PRIMER VIAJE}

El diario del primer viaje se presenta, en sus días iniciales, como un cuaderno de bitácora en el que se registran las horas de navegación y la distancia recorrida. Sin embargo, leído desde la perspectiva de Las Casas, aparece como el recorrido inicial del héroe, con sus dificultades como pruebas y con una construcción in crescendo del objeto final a descubrir. Problemas con las naves, con los marineros que lo acompañan, duración del viaje mayor a la prevista, todo lo va superando Colón con habilidad. El destino se va configurando progresivamente y estableciendo una notable tensión: ya desde el 14 de septiembre comienzan a verse indicios de la tierra; desde el 16 del mismo mes ésta se presenta con algunos de los rasgos que luego se volverán redundantes: aparece el superlativo que refiere al exceso en los rasgos eufóricos de un territorio que se percibe mediante los sentidos; éstos registran el placer que acompaña la conjunción con el valor deseado, construido según el modelo del locus amoenus del Renacimiento y asociado con el espacio más caro para el destinatario español: "Y era el tiempo como por Abril en el Andaluzía” (21) .

Una vez producido el descubrimiento, la tierra es presentada siempre de manera positiva y con rasgos que la vinculan con la representación convencional del paraíso: temperancia, abundancia, goce generado en quien lo alcanzó. Estas características positivas van aumentando en intensidad a medida que el sujeto avanza, produciendo un efecto de sentido por el cual se señala un itinerario en el que el navegante se acerca cada vez más a objetivos de perfección creciente:

Y afirma no encareçello la çentéssima parte de lo que es, y que plugo a Nuestro Señor de le mostrar siempre una cosa mejor que otra, y siempre en lo que hasta allí avía descubierto iva de bien en mejor, ansí en las tierras y arboledas

\footnotetext{
${ }^{8}$ El número entre paréntesis corresponde a la edición de Consuelo Varela que figura en la referencia bibliográfica.
} 
y yervas y frutos y flores como en las gentes, y siempre de diversa manera [...] (64).

Si bien al comienzo el término de comparación es el territorio recién reconquistado por los españoles y construido según la convención del lugar ameno, poco a poco se va aproximando a la configuración del paraíso terrenal. Primero es el nombre que le pone el Almirante el 15 de diciembre: "Puso nombre al valle Valle del Paraíso, y al río Guadalquivir, porque diz que así viene tan grande como Guadalquivir por Córdoba” (82). El nombre resulta de la observación y constatación; pero se mantiene todavía la doble referencia según la cual el territorio descubierto se parece al paraíso y también al río andaluz. La orografía descubierta se va elevando y parece alcanzar el cielo $(88,92)$, hasta que la referencia más explícita a su vinculación con el espacio de los valores máximos aparece hacia el final del viaje, el 21 de febrero:

Concluyendo, dize el Almirante que bien dixeron los sacros theólogos y los sabios philósophos que el Paraíso Terrenal esta en el fin de Oriente, porque es lugar temperadíssimo. Así que aquellas tierras que agora él avía descubierto, es -dize él- el fin del Oriente (132).

La constatación tiene la forma de reconocimiento de la coincidencia entre el discurso de los sabios y las características que Colón observa en el sitio descubierto. El Almirante ha ido avanzando en su recorrido, tanto físico como cognitivo: cada vez reconoce con mayor certeza las características excepcionales de lo que encuentra.

Esta presentación del viaje puede ser leída como resemantización de un objeto que no se ha encontrado: sobre la base del contrato inicial, las Capitulaciones de Santa Fe, Colón se ha comprometido a encontrar riquezas; no habiéndolas hallado, las reemplaza por otro bien, el paraíso que le fue dado descubrir. Sin embargo, en el planteo de Las Casas la finalidad del viaje corresponde claramente al objetivo espiritual, la conversión del indígena, el progreso de la fe y, con ello, la salvación eterna de indígenas y españoles. Construir el territorio descubierto como el paraíso, configura a Colón como el sujeto de una misión en la que está llamado a ser el héroe. El oro, según el planteo del mismo navegante, puede llegar a ser un instrumento al servicio del logro de un bien mayor: facilitar la reconquista de la Jerusalén terrestre (26 de diciembre, 101), asociada a la celestial. En este sentido, el 14 de noviembre, Colón hace referencia a los mappaemundi que, en el medievo, representaban de manera simbólica la tierra como espacio de tránsito hacia Jerusalén y, de allí, a la ciudad eterna, 
convirtiéndose así en representación de viajes espirituales (Zamora, 1993: 112 y siguientes). Las Casas silencia el objetivo primero, económico, de los viajes según las Capitulaciones, para insistir en el trascendente, de tal modo que la búsqueda de oro, desde esta perspectiva, aparece como condenada en la medida en que se convierta en la principal o, incluso, única.

Resulta particularmente significativa la insistencia de Las Casas en "dize el Almirante", por cuanto le atribuye el reconocimiento explícito de la misión encomendada que consiste en llegar al paraíso; esta meta ha sido reservada por Dios sólo para un héroe excepcional quien, ya el 23 de septiembre y en alta mar, evoca en discurso directo entrecomillado, la figura de Moisés cuando saca del cautiverio al pueblo elegido (Cf. p. 24). Colón aparece como sujeto consciente del destino que se le ha encomendado, de que ha logrado su ejecución y ha podido llegar a un espacio que termina presentándose como el paraíso terrenal. Sólo un personaje excepcional estaba en condiciones de alcanzar este fin y el relato del primer viaje culmina con un Colón-héroe sabedor de lo sucedido.

Sin embargo, mientras se van narrando los desplazamientos del Almirante por tierras caracterizadas por su excelencia, se señala también insistentemente la tensión que el sujeto manifiesta: lo percibido produce placer, se parece al paraíso, pero Colón sigue buscando otra cosa. A pesar de lo que ya encontró, el objeto de deseo es el oro que le anunciaron, las especias que aún no maduraron o las perlas que todavía no es tiempo de recoger. Aunque posea el paraíso y disfrute de él, el descubridor aspira a otro objetivo que, por el modo mismo de organizar el relato de la búsqueda de los valores, se presenta como superior para el sujeto; los bienes materiales son deseados, y por lo mismo, jerarquizados, por sobre el territorio de características excepcionales, lugar ameno primero, paraíso después.

También los indígenas son presentados como seres excepcionales. $Y$ frente a ellos, Colón es consciente de la misión encomendada a tal punto que el mismo día del descubrimiento los presenta como seres humanos a los que habrá que convertir no por ejercicio violento y unilateral del poder sino "con amor":

Yo, dize él, "porque nos tuviesen mucha amistad, porque cognosçí que era gente que mejor se libraría y convertiría a nuestra sancta fe con amor que no por fuerça [...] Ellos deven ser buenos servidores y de buen ingenio, que veo que muy presto dizen todo lo que les dezía. Y creo que ligeramente se harían cristianos, que me pareçió que ninguna secta tenían" (30-31) ${ }^{9}$.

\footnotetext{
9 Sánchez Macgrégor señala la presencia de una dimensión trascendente en los textos colombinos: "[...] a lo largo del corpus colombino abundan las imágenes fideístas (fe religiosa) que 'materializan' o concretan los códigos salvador, utopista, de lo absoluto [...]” (1991: 55).
} 
Nuevamente se pone en boca de Colón las palabras mediante las cuales reconoce el objetivo del viaje y la manera de realizarlo, teniendo en cuenta las características de los seres humanos que se le presentan. Como afirma Margarita Zamora, "La intervención metalingüística 'dize el Almirante' marca el comienzo exacto del testimonio sobre la naturaleza moral idealizada del indígena como creatura que vive en una edad de oro moral- estado que, dice Colón, es especialmente apto para la implantación de la fe cristiana" (1993: 45). El indígena, definido por las carencias - no tiene ropa, ni armas, ni secta-, aparece como el hombre natural, de "buen ingenio", susceptible de convertirse en vasallo de la corona y cristiano, "con amor que no por fuerça". Resulta llamativa la equivalencia entre las palabras del Almirante, puestas directamente en su boca, y la misión que le atribuye Las Casas en la Historia de las Indias: Colón es presentado como sujeto consciente de lo que debe hacer. $Y$ esa conciencia se hace más explícita si tenemos en cuenta que Las Casas le atribuye el discurso directo en situaciones predominantemente vinculadas al reconocimiento de la misión y de su factibilidad, ya sea porque Dios lo acompaña, ya sea porque la tierra y los hombres son excelentes. Hasta el mar es calmo y no hay tormentas; el mal tiempo lo sorprende recién cuando está llegando al territorio europeo:

Dize que estava maravillado de tan mal tiempo como avía en aquellas islas y partes, porque en las Indias navegó todo aquel invierno sin surgir, e avía siempre buenos tiempos, y que una sola ora no vido la mar que no se pudiese bien navegar [...] (132).

En este mismo sentido, puede leerse el hecho de que Colón se convierta, desde el primer día del encuentro con el nativo, en el enunciador de la norma. Ya el 13 de octubre Colón afirma: "Esto [el excesivo desequilibrio en el trueque] defendiera y no dexara tomar a nadie salvo que yo lo mandara tomar todo para Vuestras Altezas" (32). Sin embargo, ese mismo día, "[...] yo estava atento y trabajava de saber si avía oro [...] Y también aquí naçe el oro que traen colgado a la nariz, mas, por no perder tiempo, quiero ir a ver si puedo topar a la isla de Çipango" (32). "Son estas islas muy verdes y fértiles y de aires muy dulçes, y puede aver muchas cosas que yo no sé, porque no me quiero detener por calar y andar muchas islas para fallar oro" (35). El texto registra de la tensión de quien subestima la excelencia de hombres y tierra, pospone su misión transformadora, para aspirar a algo que, para él, es más importante: el oro. Las mismas palabras del Almirante permiten configurar su culpa. En numerosas oportunidades, Colón se refiere a la intervención divina, sobre todo al final, cuando dice: "esto d'este viaje cognosco", dize el Almirante, "que milagrosamente lo a 
mostrado[s], así como se puede comprehender por esta escriptura, por muchos milagros señalados amostrado<s> en el viaje" (138). Si es consciente de la intervención divina, la reconoce y declara, la sustitución de los valores trascendentes por los materiales constituye, en el planteo lascasiano, un pecado.

En la presentación del nativo, el discurso de Colón da cuenta de la misma dualidad que aparece en el texto de Las Casas: los indígenas son definidos por la falta de ropa, armas y sectas, pero esas carencias los aproximan al hombre natural, habitante del paraíso previo al pecado ${ }^{10}$. Su belleza es llamativa, ajena al paso del tiempo -Colón no ve indígenas de más de treinta años ${ }^{11}$ - y están dispuestos para la transformación. Su generosidad se opone a la codicia de los españoles en una llamativa forma de designación del pecado capital cometido por los descubridores que coincide con el que les atribuye Las Casas. Además, los indígenas, antes de la conversión misma, cumplen con el mandamiento de los cristianos, una vez más en palabras del Almirante:

[...] dize el Almirante, "son gente de amor y sin cudiçia y convenibles para toda cosa, que certifico a Vuestras Altezas que en el mundo creo que no ay mejor gente ni mejor tierra. Ellos aman a sus próximos como a sí mismos, y tienen una habla la más dulçe del mundo, y mansa y siempre con risa” (98).

Este hombre similar al Adán del paraíso demuestra, al mismo tiempo, su capacidad de ejercer el control sobre la naturaleza. Aunque ésta produzca sin necesidad de mucho trabajo -recordemos que éste, acompañado del sudor de la frente, es consecuencia del castigo por el pecado original-, los que ve Colón son tierras labradas, productivas, y pueblos organizados, regidos por reyes caracterizados positivamente:

"[...] y las cosas y lugares tan hermosos, y con señorío en todos, como juez o señor d'ellos, y todos le obedeçen que es maravilla, y todos estos señores son de pocas palabras y muy lindas costumbres, y su mando es lo más con hazer señas con la mano, y luego es entendido que es maravilla". Todas son palabras del Almirante. (97)

"[...] entre sí tienen costumbres muy buenas, y el rey muy maravilloso estado, de una cierta manera tan continente qu'es plazer de verlo todo" (98).

10 "Los amerindios, por su parte, fueron descritos por Colón como hombres naturales que llevaban una vida armoniosa en comunión con una naturaleza de fauna abundante, flora exótica y ricos yacimientos de oro" (González Ortega, 2006: 138).

${ }^{11}$ Aunque Las Casas ironice con respecto a la incorrecta "visión" del Almirante, importa esta caracterización positiva del indígena. 
En discurso directo del Almirante, aparecen los rasgos que definen la humanidad del indígena de acuerdo a la propuesta lascasiana: capacidad de control de la naturaleza para su propio beneficio y dominio de la naturaleza humana para hacer posible la convivencia. Sus casas son limpias y ordenadas, tienen monedas con inscripciones -comercio y escritura-, e incluso estatuas. Hombres inscriptos en el marco de la ley natural, dispuestos para el cambio mejorador por la conversión al cristianismo, sus conductas los muestran como seres racionales y anticipan la puesta en acto de los preceptos cristianos. El primer conflicto con los indígenas se produce el 13 de enero, después de haber hecho referencia, el 6, 8 y 10 de enero, a la codicia de oro de Martín Alonso Pinzón, a las obras de Satanás (109) y a la maldad "que era cosa tan pública [...] que no se podía encobrir" (112). Sin que podamos hablar de una relación de causa a efecto entre la culpa de uno de los Pinzones y el enfrentamiento con los indígenas, resulta curioso el orden en que se presentan los episodios, acorde a la causalidad establecida por Las Casas en su Historia.

\subsection{RELATO DEL TERCER Y CUARTO VIAJES}

En el relato del tercer viaje se sintetizan y afianzan los rasgos fundamentales que acabamos de señalar en el primero. Colón se presenta como ejecutor de un destino ya profetizado por Isaías (205) que lo lleva al paraíso terrenal, prefigurado por los teólogos (218). Esa misión ha sido confiada por el mismo Dios a un ser predestinado: "[...] creo que allí es el Paraíso Terrenal, adonde no puede llegar nadie salvo por voluntad divina” (218). El éxito adquiere dos dimensiones: por una parte, se confirma la hipótesis geográfica según la cual la tierra sería redonda, con una pequeña variante que sin desdecir la primera, refuerza la conformación del objeto de deseo sobre la dimensión erótica; por otra, lo descubierto es el paraíso terrenal, premio mayor de la hazaña.

Los objetivos del viaje reaparecen en apretado resumen: servicio a Dios y a la Corona; éstos son tanto políticos, sobre todo en relación a Portugal, como trascendentes ya que se garantiza la ampliación del reino y la expansión de la fe. Y también se menciona el objetivo económico, aunque aquí se señalen las ventajas para los reyes y no el beneficio personal: no se ha enviado tanto oro como se esperaba, pero "se an enbiado suffiçientes muestras d'ello y de otras cosas de valor" (220). El héroe ha sido premiado, ha debido enfrentar a enemigos, pero cuenta con el apoyo de sus destinadores iniciales.

Del cuarto viaje sólo se conserva copia de una supuesta carta escrita desde Jamaica en julio de 1503. La relación con la Historia de las Indias de Las Casas no es la misma que en los textos anteriores: no se trata de una transcrip- 
ción, aunque el dominico mencione fragmentos de un presunto original. Por lo mismo, no es posible sostener sin reparos la hipótesis de la proyección de la intencionalidad lascasiana sobre las operaciones de selección, énfasis y organización de los originales colombinos. Sin embargo, no podemos dejar de señalar algunos aspectos que nos parecen significativos.

Ante todo, si el recorrido del héroe ha venido siendo presentado por Las Casas como un viaje de sentido espiritual que culmina con el descubrimiento del paraíso; si el destino ha sido parcialmente realizado en tanto se efectuó con éxito el desplazamiento del sujeto hacia el objeto descripto en términos superlativos, pero a la vez, el personaje se ha desviado del mandato sustituyendo la salvación eterna por la búsqueda del oro, el proceso de reconocimiento del héroe plantea una disyuntiva: merece, a la vez, el premio y el castigo. La solución la ofrece el mismo Las Casas en un texto que da cuenta de una concepción cristiana -al menos en la época- de la justicia divina: el Almirante merece la salvación eterna por el descubrimiento, pero la culpa ha de ser redimida mediante el sufrimiento en la tierra:

[...] todos estos infortunios y adversidades, angustias y penalidades fueron de aquellas culpas el pago y castigo [...] A la bondad y misericordia de Dios plega de contentarse, rescibiendo por satisfacción de las culpas que en estas tierras que descubrió contrajo, las tribulaciones, angustias y amarguras, con los peligros, trabajos y sudores que toda su vida padesció, porque en la otra vida le haya concedido perpetuo descanso (Lib. II, Cap. XXXVIII, T. II, 330 - 331).

Las Casas, en su relato del último viaje, acumula desgracias: enfermedad, sublevación de los marineros, conflicto con los indígenas, tormentas, sobre todo tormentas... Por su parte, Colón -siempre y cuando sigamos creyendo que es el autor de los textos que se le atribuyen- consigna tormentas extremas, al punto de vincularlas con el fin del mundo, el diluvio universal y el mismo infierno en el modo de representación del mar embravecido. Luego se le presenta Dios, le recuerda la misión encomendada y lo induce a reconocer el yerro. "Yo, assí amortecido, oí todo, mas no tuve yo respuesta a palabras tan ciertas, salvo llorar por mis yerros. Acabó el de fablar, quienquiera que fuese, diciendo: 'No temas, confía: todas estas tribulaciones están escritas en piedra mármol y no sin causa'" (298).

Los suyos lo han abandonado y pesa sobre él la calificación de extranjero. Las mujeres son aquí "putas" (300) y los indígenas sus enemigos:

Yo estoy tan perdido como dixe. Yo he llorado fasta aquí a otros. Aya missericordia agora el cielo y llore por mí la tierra. En el temporal no tengo solamen- 
te una blanca para el oferta, en el espiritual he parado aquí en las Indias de la forma que está dicho: aislado en esta pena, enfermo, aguardando cada día por la muerte y cercado de un cuento de salvajes y llenos de crueldad y enemigos nuestros, y tan apartado de los santos Sacramentos de la Sancta Iglesia, que se olvidará d'esta ánima si se aparta acá del cuerpo. Llore por mí quien tiene caridad, verdad y justiçia (304).

La alusión al oro, en este contexto, parece un recordatorio de la culpa: "El oro es excelentíssimo; del oro se hace tesoro, y con él, quien lo tiene, haçe cuanto quiere en el mundo, y llega a que echa las ánimas al Paraíso" (302). Cabe pensar en que el oro puede ser bien usado para objetivos superiores, vinculando el negocio material con el negotium crucis (cf. Zamora 1993:), pero nuevamente llama la atención el modo como se parecen los relatos de Colón y Las Casas: la evocación de la riqueza se yuxtapone a las alusiones al sufrimiento como castigo temporal que evita la pena eterna.

\section{A MODO DE CIERRE...}

No podemos negar del todo la posible transcripción literal de los textos colombinos por parte de Bartolomé de Las Casas. Puede pensarse que lo escrito por el Almirante le sirvió al dominico para proponer su versión de la historia. Sin embargo, el uso selectivo de la palabra directa; la necesaria jerarquización de los datos en las opciones que exige la síntesis; la redundancia en las operaciones de yuxtaposición en los relatos del descubrimiento de las alusiones al paraíso y a la búsqueda del oro; la organización global de los viajes que siguen un orden en el que se suceden el reconocimiento de la misión, su progresivo cumplimiento, el éxito, la culpa y las compensaciones que salvaguardan el premio eterno, parecen más propios del planteo de un religioso, sacerdote y juez que transcribe el texto de otro en función de sus objetivos. Visto de esta manera, el discurso de Colón parece más bien la prueba -recortada, resumida, re-escrita- que funda el juicio de Las Casas.

\section{REFERENCIAS}

Carpentier, Alejo. 1979. El arpa y la sombra. Madrid: Siglo XXI Editores. Casas, Bartolomé de las. 1981. Historia de las Indias (3 vols). México: Fondo de Cultura Económica. 
Colón, Cristóbal. 1982. Textos y documentos completos. Prólogo y notas de Consuelo Varela: Madrid: Alianza Editorial.

González Ortega, Nelson. 2006. Relatos mágicos en cuestión. La cuestión de la palabra indigena, la escritura imperial y las narrativas totalizadoras y disidentes de Hispanoamérica. Madrid: Iberoamericana-Vervuert.

Jitrik, Noé. 1992. Historia de una mirada. El signo de la cruz en las escrituras de Colón. Buenos Aires: Ediciones de la Flor.

O'Gorman, Edmundo. 1984. La invención de América. México: Fondo de Cultura Económica.

Pastor, Beatriz. 1988. Discursos narrativos de la conquista: mitificación y emergencia. Hanover: Ediciones del Norte.

Roa Bastos, Augusto.1992. Vigilia del almirante. Buenos Aires: Sudamericana. Sánchez Macgrégor, Joaquín. 1991. Colón y Las Casas. Poder y contrapoder en la filosofía de la historia latinoamericana. México D.F.: Universidad Nacional Autónoma de México.

Todorov, Tzvetan. 1987. La conquista de América. El problema del otro. México: D.F. Siglo Veintiuno Editores.

Zamora, Margarita. 1993. Reading Columbus. Los Angeles-London: University of California Press Berkeley. 